\title{
Pathogenic and Diagnostic Potential of BLCA-1 and BLCA-4 Nuclear Proteins in Urothelial Cell Carcinoma of Human Bladder
}

\author{
Matteo Santoni, ${ }^{1}$ Francesco Catanzariti, ${ }^{2}$ Daniele Minardi, ${ }^{2}$ Luciano Burattini, ${ }^{1}$ \\ Massimo Nabissi, ${ }^{3}$ Giovanni Muzzonigro, ${ }^{2}$ Stefano Cascinu, ${ }^{1}$ and Giorgio Santoni ${ }^{3}$ \\ ${ }^{1}$ Department of Medical Oncology, Polytechnic University of the Marche Region, 60126 Ancona, Italy \\ ${ }^{2}$ Division of Urology, Department of Clinical and Specialist Sciences, Polytechnic University of the Marche Region, \\ 60126 Ancona, Italy \\ ${ }^{3}$ Section of Experimental Medicine, School of Pharmacy, University of Camerino, Via Madonna delle Carceri, \\ 62032 Camerino, Italy \\ Correspondence should be addressed to Giorgio Santoni, giorgio.santoni@unicam.it
}

Received 3 March 2012; Revised 13 May 2012; Accepted 1 June 2012

Academic Editor: Martin Schostak

Copyright (C) 2012 Matteo Santoni et al. This is an open access article distributed under the Creative Commons Attribution License, which permits unrestricted use, distribution, and reproduction in any medium, provided the original work is properly cited.

\begin{abstract}
Transitional cell carcinoma (TCC) of the bladder is one of the most common malignancies of genitourinary tract. Patients with bladder cancer need a life-long surveillance, directly due to the relatively high recurrence rate of this tumor. The use of cystoscopy represents the gold standard for the followup of previously treated patients. Nevertheless, several factors, including cost and invasiveness, render cystoscopy not ideal for routine controls. Advances in the identification of specific alterations in the nuclear structure of bladder cancer cells have opened novel diagnostic landscapes. The members of nuclear matrix protein family BLCA-1 and BLCA-4, are currently under evaluation as bladder cancer urinary markers. They are involved in tumour cell proliferation, survival, and angiogenesis. In this paper, we illustrate the role of BLCA-1 and BLCA-4 in bladder carcinogenesis and their potential exploitation as biomarkers in this cancer.
\end{abstract}

\section{Background}

Transitional cell carcinoma (TCC) represents more than $90 \%$ of bladder cancers [1], ranking among genitourinary malignancies only behind prostate cancer for frequency and estimated mortality. At initial diagnosis, more than $70 \%$ of bladder tumors are confined to the mucosa or lamina propria. Transurethral resection of nonmuscle invasive tumors can be accompanied by intrabladder therapy, depending on tumor depth and grade. However, more then $70 \%$ of patients can present tumor recurrences after treatment, with up to $30 \%$ of patients progressing to higher tumor stage and grade [2].

In this view, close and accurate disease surveillance is essential for monitoring tumour recurrence and progression to invasive disease. The current standard diagnostic iter includes urine cytology, imaging, and flexible cystoscopy. Cytology represents the cornerstone of urine-based bladder cancer diagnosis. It involves microscopic examination of precancerous and cancerous cells present in the urine by a pathologist. Although its high specificity $(96 \%)$, the sensitivity is lower (44\%) [3], particularly for low-grade tumors [4].

Quanticyt is a karyometric of bladder washing for the quantitative grading of urine cytology [5]. Based on the DNA content levels and nuclear morphometry, bladder cancer can be classified into low, intermediate, and high risk of recurrence [6]. Emerging data from the main studies involving the use of Quanticyt showed that this test has a sensitivity of $56.4 \%$ (range $42.1-69 \%$ ) and a specificity of $72.1 \%$ (range $67.9-76 \%)[7,8]$.

The use of cystoscopy has been successful in monitoring bladder cancer recurrence [9]. On the other hand, cystoscopy is not ideal for the life-long followup of patients with bladder cancer, considering its cost and invasiveness; moreover, the difficulty in identifying asymptomatic patients has prompted 
the search for more reliable noninvasive markers for the early detection of bladder cancer.

Noninvasive urine-based markers represent a novel diagnostic approach. BCLA- 1 and BCLA- 4 are included in this list that also comprises nuclear matrix protein 22 (NMP22) and bladder tumour antigen (BTA).

In 1996, Getzenberg et al. identified six bladder-specific nuclear structure proteins (BLCA-1 to 6), expressed exclusively by bladder cancer cells [10]. These nuclear matrix proteins (NMPs) are involved in several functions, including DNA replication, RNA synthesis, and nuclear morphology. This review describes the functional role played by BLCA- 1 and BLCA-4 in bladder carcinogenesis, illustrating the currently available data concerning their potential diagnostic employ.

\section{Functional Role of BLCA-1 and BLCA-4 in Bladder Carcinogenesis}

Changes in nuclear structure can affect gene expression, thus playing an important role in the carcinogenesis process [11]. In 1977, Berezney and Coffey first described the nuclear matrix structure [12]. It is composed by protein components derived from three structural regions: a lamina with nuclear pores, the residual nucleoli, and an internal matrix framework connected to a residual nuclear layer containing pore complexes. Nuclear matrix represents an active environment where DNA replication $[13,14]$ and RNA synthesis take place $[15,16]$. NMPs recognize and bind to specific DNA sequences called scaffold/matrix attachment regions (S/MAR), partitioning DNA into functional loop domains. S/MARs are involved in chromosomal replication, transcription, recombination, and condensation. They interact with topoisomerase II, identified by Berrios et al. in 1985 as a major polypeptide component of the Drosophila nuclear matrix-pore complex-lamina fraction [17]. The S/MAR interacting elements also include lamins A and C [18], Poly (ADP-ribose)polymerase 1 and 2 (PARP-1, PARP-2) [19], and CCCTC-binding factor (CTCF) [20] that binds to the regulatory regions of $c-m y c$ gene [21]. Moreover, certain $\mathrm{S} / \mathrm{MARs}$ require adjacent transcription factors to become active [22]. Therefore, nuclear morphology is deeply influenced by NMPs. Based on these findings, NMPs have been investigated as potential cancer markers. Moreover, the discovery that NMPs are released into urine and serum has suggested their exploitation for cancer diagnosis.

NMP-22 (NuMA) is a $239 \mathrm{kDa}$ nuclear matrix protein located in the mitotic spindle [23]. It is involved in microtubule assembly and in the partitioning of genome into newly formed G1 nuclei during cellular replication [24]. NMP22 is released from cells undergoing apoptosis [25]. NMP22 levels are higher in bladder cancer cells as compared to the normal counterpart [26]. NMP22 test has been the first nuclear matrix-based test approved by the U.S. Food and Drug Administration (FDA) for the followup of bladder cancer patients and for the screening of patients with suspected symptoms or with a family history of bladder cancer. Sensitivity of NMP22 test varies from $68.5 \%$ to $88.5 \%$ and specificity varies from $65.2 \%$ to $91.3 \%$ depending on the chosen cut-off [27-32]. Positive and negative predictive values of NMP 22 test vary from $34 \%$ to $76 \%$ and from $77.9 \%$ to $98 \%$, respectively [28-31].

Evaluation of NMP22 has been also used for the diagnosis of colorectal carcinoma [33] and as a rejection marker in kidney transplantation [34].

BLCA-1 was originally identified from bladder tumor tissue [10]. While BLCA- 4 is detected also in normal adjacent tissue, BLCA-1 is only expressed by tumor cells, suggesting their different role in bladder carcinogenesis.

BLCA-1 gene is similar to TI-227H, a cancer metastasisassociated gene discovered in 1996 by Ishiguro et al. [35] and isolated from the B16-F10 mouse melanoma subline [36]. The homology with TI-227H gene suggested a potential role of BLCA-1 to discriminate metastatic versus localized disease. However, no relation has been found so far between BLCA-1 levels and advanced bladder cancer.

In 2005, Myers-Irvin et al. [37] examined BLCA-1 expression in bladder tissue and urine via Western blot and ELISA, respectively. They observed that BLCA-1 protein levels were significantly higher in patients with bladder cancer than in normal individuals but did not correlate with tumor grade. They registered $80 \%$ sensitivity and $87 \%$ specificity, demonstrating the potential employment of a BLCA-1-based assay in diagnosis and surveillance of patients with bladder cancer.

The gene that encodes BLCA- 4 was sequenced by Getzenberg et al. in 2004. They observed common regions (a nuclear localization sequence and helix-loop-helix region) with the ELK-3 gene, a member of the ETS transcription family [38]. These factors bind to DNA, specifically interacting with C/A GGA A/T sequences. ETS transcription factors are involved in apoptosis, carcinogenesis, VEGF expression, and angiogenesis, and local and metastatic diffusion [39].

Elk-3 is phosphorylated and activated by the Rasextracellular signal-regulated kinase (Erk) pathway. Together with Elk-1 and Sap-1, Elk-3 forms the ternary complex transcription factor subfamily (TCF), which regulates the early response of quiescent cells to growth factor stimulation [40]. BLCA-4 interacts with several known transcription factors, such as AP-1, AP-2, NFATc, NF-E1, and NF-E2 [38]. In 2005, Myers-Irvin et al. analyzed the BLCA- 4 functions by transfecting the BLCA-4 gene into four different human bladder carcinoma cell lines. BLCA-4-transfected bladder cancer cells exhibited a growth advantage as compared to untransfected cells. Additionally, microarray analysis revealed an upregulation of genes related to cell growth, including the cyclins, as well as of interleukin-8 (IL-8), thrombomodulin (TM), and interleukin-1alpha (IL- $1 \alpha)$ in overexpressing BLCA-4 cells. Analysis of the $I L-1 \alpha, I L-8$, and TM gene promoters evidenced multiple ETS sites in their sequence, suggesting that BLCA-4 binds directly to each gene to cause overexpression [41].

IL-8 has been shown to play an important role in multiple cellular processes. In 1997, Thalmann et al. observed that urinary IL-8 was a prognostic factor of bladder cancer recurrence and progression after Bacillus Calmette-Guerin therapy [42]. In 2002, Lang et al. described the signaling 
pathway regulating IL-8-supported bladder carcinoma cell migration. In 2008, Chikazawa et al. observed in an orthotopic model of murine bladder cancer that tumorigenicity, angiogenesis, and metastasis formation were significantly higher for tumor cells expressing higher IL-8 levels [43]. This increased tumor growth and metastasis formation could be attributed to the IL-8-mediated up-regulation of the metalloproteinases MMP-2 and MMP-9 expression and activity [44]. In 2009, Tseng-Rogenski and Liebert suggested an additional role for IL-8 as a growth and essential survival factor for normal human urothelial cells. They observed that exogenous human recombinant IL-8 promoted normal urothelial cell growth through the activation of Akt pathway [45].

The important role of IL-8 in bladder cancer is also suggested by the results obtained by Milan et al. [44]. They tested the ability of a fully human anti-IL-8 antibody, ABXIL8, to affect TCC growth in vitro and in an in vivo mouse model. They observed a significant decrease in tumor growth accompanied by downmodulation of the nuclear factorkappaB (NF- $\kappa \mathrm{B})$ expression and transcriptional activity, and consequently in significant inhibition of MMP-2 and MMP-9 expression.

IL-8-251 T > A polymorphism also seems to be a relevant susceptibility factor for bladder carcinoma development and to influence bladder cancer patients' outcome after BCG immunotherapy [46].

Finally, recent studies suggest a potential role of IL-8 in malignant transformation of urothelial cells $[47,48]$.

IL-1 is an inflammatory cytokine, present in two different isoforms, IL- $1 \alpha$ and IL- $1 \beta$, with similar biological functions. The role of IL- 1 in bladder cancer tumorigenesis and angiogenesis is still unclear. IL-1 can promote bladder cancer cell adhesion and increase the expression of matrix degrading enzymes, thus favoring tumor invasion. However, IL-1 $\alpha$ has also been reported to reduce tumor angiogenesis and participate in the regulation of immune responses. These data were confirmed by Seddighzadeh et al., who observed a correlation between low IL-1 $\alpha$ messenger RNA levels and decreased overall survival (OS) in patients with urinary bladder carcinoma [49].

TM is a cell-surface-expressed glycoprotein, predominantly synthesized by vascular endothelial cells [50]. TM is a necessary anticoagulant factor in the protein $\mathrm{C}$ pathway and is also involved in inflammation, fibrinolysis, apoptosis, cell adhesion, and cellular proliferation [51-53]. BLCA-4 over-expression results in increased TM expression, which sustains the microcirculation [54] needed for tumor cell survival.

In 2000, Konety et al. [55] detected 53 out of 55 samples of histology proven bladder carcinoma cases through the high level of BLCA4 in urine, without registering any false positivity in 51 normal controls. In addition, in 2005 Van Le et al. [56] developed a sandwich immunoassay based on two BLCA-4 antibodies directed against distinct epitopes. BLCA-4 was measured in precipitated urine samples from patients divided into four different groups. Group A consisted of patients with bladder cancer, groups B and C included patients with various benign urologic conditions, group D was composed by patients with prostate cancer, and group E with healthy individuals. The mean BLCA-4 level for the patients in Group A was significantly higher than the mean of the other groups. Thus, the indirect BLCA-4 immunoassay showed a specificity of $95 \%$ and a sensitivity of $89 \%$.

\section{Conclusion}

Bladder cancer is the fourth most common cancer in men and the ninth most common in women. Despite recent advances, the molecular mechanisms underlying bladder carcinogenesis are still not fully elucidated so far. The screening of high-risk population has become critical to diminish the mortality. Current methods used for a proper diagnosis of bladder cancer mainly rely on cystoscopy, which can be associated with biopsy or resection. The importance of detecting bladder cancer at the early stage is clearly demonstrated by the $94 \%$ of 5 -year survival rate registered in patients with localized disease. Cytology has represented for decades the noninvasive standard for the detection of urinary bladder cancer cells. However, cytology lacks sensitivity, particularly for low-grade tumors. In the last years, the list of identified urinary markers in course of evaluation has been rapidly enlarged. Preliminary data on emerging markers have shown higher sensitivity than routine urinary cytology, especially when a panel of markers were used. The urinary markers and tests under investigation include BTA, NMP22, BLCA-1, BLCA-4, hyaluronic acid, hyaluronidase, cytokeratin-8, cytokeratin-18, cytokeratin-19, telomerase, Immunocyt, Quanticyt, FDP, FISH, and CYFRA-21-1.

At present, the role of BLCA-1 in bladder carcinogenesis has not been clarified. The homology with the TI-227H metastasis-associated gene suggests BLCA- 1 a potential candidate to discriminate metastatic versus localized disease. The use of BLCA-1 as urinary marker of bladder cancer has provided promising results, being observed a sensitivity and a specificity of $80 \%$ and $87 \%$, respectively. Moreover, further studies are required to investigate the correlation of BLCA-1 expression with tumor grade and clinical stage, considering the small number of low-grade tumors in the study by MyersIrvin et al.

As regards to BLCA-4, results show that its expression does not only provide bladder cancer cells with growth advantage but can also cause malignant cell transformation. BLCA- 4 activity is mediated by IL-1, IL- 8 , and thrombomodulin, which can act by maintaining blood flow for tumor cell survival, enhancing tumor cell proliferation and invasion, and increasing tumor angiogenesis.

Among urinary markers for bladder cancer, BLCA- 4 has registered the highest sensitivity and specificity. Its potential is further strengthened by the absence of high BLCA- 4 levels in patients with various benign urologic disorders, such as urinary tract infection, catheterization, or cystitis. Nevertheless, novel methods not requiring urine precipitation analysis are necessary to include BLCA- 4 assay into clinical practice.

In conclusion, preliminary data suggest that BLCA-1 and BLCA-4 show a high potential as bladder cancer urinary markers. Their employ may aid to identify individuals in the early stages of bladder cancer, thus affecting the therapeutic approach and prognosis of these patients. Further studies 
are required to evaluate the role of the BLCA-1 and BLCA4 assays in the detection of this disease.

\section{Conflict of Interests}

The authors declare to have no conflict of interests.

\section{Acknowledgments}

Sincere gratitude is owned to the supervisors, Dr. Rossana Berardi and Dr. Mario Scartozzi, for their support throughout this work. Particular thanks are due to Dr. Luciano Burattini for his precious endorsement.

\section{References}

[1] S. Al-Sukhun and M. Hussain, "Molecular biology of transitional cell carcinoma," Critical Reviews in Oncology/Hematology, vol. 47, no. 2, pp. 181-193, 2003.

[2] C. Kwak, J. H. Ku, J. Y. Park, E. Lee, S. E. Lee, and C. Lee, "Initial tumor stage and grade as a predictive factor for recurrence in patients with stage T1 grade 3 bladder cancer," Journal of Urology, vol. 171, no. 1, pp. 149-152, 2004.

[3] G. Mowatt, S. Zhu, M. Kilonzo et al., "Systematic review of the clinical effectiveness and cost-effectiveness of photodynamic diagnosis and urine biomarkers (FISH, ImmunoCyt, NMP22) and cytology for the detection and follow-up of bladder cancer," Health Technology Assessment, vol. 14, no. 4, pp. 1-331, 2010.

[4] D. M. Schwalb, H. W. Herr, and W. R. Fair, "The management of clinically unconfirmed positive urinary cytology," Journal of Urology, vol. 150, no. 6, pp. 1751-1756, 1993.

[5] H. G. Van der Poel, M. R. Van Balken, D. H. J. Schamhart et al., "Bladder wash cytology, quantitative cytology, and the qualitative BTA test in patients with superficial bladder cancer," Urology, vol. 51, no. 1, pp. 44-50, 1998.

[6] H. G. Van der Poel, J. A. Witjes, P. Van Stratum, M. E. Boon, F. M. J. Debruyne, and J. A. Schalken, "Quanticyt: karyometric analysis of bladder washing for patients with superficial bladder cancer," Urology, vol. 48, no. 3, pp. 357-364, 1996.

[7] P. M. J. Moonen, G. F. M. Merkx, P. Peelen, H. F. M. Karthaus, D. F. C. M. Smeets, and J. A. Witjes, "UroVysion compared with cytology and quantitative cytology in the surveillance of non-muscle-invasive bladder cancer," European Urology, vol. 51, no. 5, pp. 1275-1280, 2007.

[8] B. W. G. Van Rhijn, H. G. Van der Poel, and T. H. Van der Kwast, "Urine markers for bladder cancer surveillance: a systematic review," European Urology, vol. 47, no. 6, pp. 736-748, 2005.

[9] P. Whelan, J. P. Britton, and A. C. Dowell, "Three-year followup of bladder tumours found on screening," British Journal of Urology, vol. 72, no. 6, pp. 893-896, 1993.

[10] R. H. Getzenberg, B. R. Konety, T. A. Oeler et al., "Bladder cancer-associated nuclear matrix proteins," Cancer Research, vol. 56, no. 7, pp. 1690-1694, 1996.

[11] B. R. Konety and R. H. Getzenberg, "Nuclear structural proteins as biomarkers of cancer," Journal of Cellular Biochemistry, vol. 76, no. 33, pp. 183-191, 1999.

[12] R. Berezney and D. S. Coffey, "Nuclear matrix. Isolation and characterization of a framework structure from rat liver nuclei," Journal of Cell Biology, vol. 73, no. 3, pp. 616-637, 1977.
[13] S. J. McCready, J. Godwin, and D. W. Mason, "DNA is replicated at the nuclear cage," Journal of Cell Science, vol. 46, pp. 365-386, 1980.

[14] R. Berezney and L. A. Buchholtz, "Dynamic association of replicating DNA fragments with the nuclear matrix of regenerating liver," Experimental Cell Research, vol. 132, no. 1, pp. $1-13,1981$.

[15] E. M. Ciejek, J. L. Nordstrom, M. J. Tsai, and B. W. O’Malley, "Ribonucleic acid precursors are associated with the chick oviduct nuclear matrix," Biochemistry, vol. 21, no. 20, pp. 4945-4953, 1982.

[16] C. A. G. van Eekelen and W. J. Van Venrooij, "hnRNA and its attachment to a nuclear protein matrix," Journal of Cell Bio$\log y$, vol. 88, no. 3, pp. 554-563, 1981.

[17] M. Berrios, N. Osheroff, and P. A. Fisher, "In situ localization of DNA topoisomerase II, a major polypeptide component of the Drosophila nuclear matrix fraction," Proceedings of the National Academy of Sciences of the United States of America, vol. 82, no. 12, pp. 4142-4146, 1985.

[18] D. J. Hakes and R. Berezney, "DNA binding properties of the nuclear matrix and individual nuclear matrix proteins: evidence for salt-resistant DNA binding sites," Journal of Biological Chemistry, vol. 266, no. 17, pp. 11131-11140, 1991.

[19] F. Tramontano, S. Di Meglio, and P. Quesada, "Co-localization of poly(ADPR)polymerase 1 (PARP-1) poly(ADPR)polymerase 2 (PARP-2) and related proteins in rat testis nuclear matrix defined by chemical cross-linking," Journal of Cellular Biochemistry, vol. 94, no. 1, pp. 58-66, 2005.

[20] K. L. Dunn and J. R. Davie, "The many roles of the transcriptional regulator CTCF," Biochemistry and Cell Biology, vol. 81, no. 3, pp. 161-167, 2003.

[21] E. M. Klenova, R. H. Nicolas, H. F. Paterson et al., "CTCF, a conserved nuclear factor required for optimal transcriptional activity of the chicken c-myc gene, is an 11-Zn-finger protein differentially expressed in multiple forms," Molecular and Cellular Biology, vol. 13, no. 12, pp. 7612-7624, 1993.

[22] M. Klar, E. Stellamanns, P. Ak, A. Gluch, and J. Bode, "Dominant genomic structures: detection and potential signal functions in the interferon-beta domain," Gene, vol. 364, no. 1-2, pp. 79-89, 2005.

[23] C. H. Yang, E. J. Lambie, and M. Snyder, "NuMA: an unusually long coiled-coil related protein in the mammalian nucleus," Journal of Cell Biology, vol. 116, no. 6, pp. 1303-1317, 1992.

[24] M. A. Mancini, D. He, I. I. Ouspenski et al., "Dynamic continuity of nuclear and mitotic matrix proteins in the cell cycle," Journal of Cellular Biochemistry, vol. 62, pp. 158-164, 1996.

[25] M. Hoffman, “The cell's nucleus shapes up," Science, vol. 259, no. 5099, pp. 1257-1259, 1993.

[26] S. K. Keesee, J. V. Briggman, G. Thill, and Y. J. Wu, "Utilization of nuclear matrix proteins for cancer diagnosis," Critical Reviews in Eukaryotic Gene Expression, vol. 6, no. 2-3, pp. 189214, 1996.

[27] J. Landman, Y. Chang, E. Kavaler, M. J. Droller, and B. C. S. Liu, "Sensitivity and specificity of NMP-22, telomerase, and BTA in the detection of human bladder cancer," Urology, vol. 52, no. 3, pp. 398-402, 1998.

[28] V. Poulakis, U. Witzsch, R. De Vries, H. M. Altmannsberger, M. J. Manyak, and E. Becht, "A comparison of urinary nuclear matrix protein-22 and bladder tumour antigen tests with voided urinary cytology in detecting and following bladder cancer: the prognostic value of false-positive results," $B J U$ International, vol. 88, no. 7, pp. 692-701, 2001.

[29] S. Eissa, M. Swellam, M. Sadek et al., "Comparative evaluation of the nuclear matrix protein, fibronectin, urinary bladder 
cancer antigen and voided urine cytology in the detection of bladder tumors," Journal of Urology, vol. 168, no. 2, pp. 465469, 2002.

[30] M. G. Friedrich, A. Hellstern, S. H. Hautmann et al., "Clinical use of urinary markers for the detection and prognosis of bladder carcinoma: a comparison of immunocytology with monoclonal antibodies against Lewis X and 486p3/12 with the BTA Stat and NMP22 tests," Journal of Urology, vol. 168, no. 2, pp. 470-474, 2002.

[31] L. E. Ponsky, S. Sharma, L. Pandrangi et al., "Screening and monitoring for bladder cancer: refining the use of NMP22," Journal of Urology, vol. 166, no. 1, pp. 75-78, 2001.

[32] S. Ramakumar, J. Bhuiyan, J. A. Besse et al., "Comparison of screening methods in the detection of bladder cancer," Journal of Urology, vol. 161, no. 2, pp. 388-394, 1999.

[33] U. Hasholzner, P. Stieber, A. Zimmermann et al., "Nuclear mitotic apparatus protein (NuMA) in benign and malignant diseases," Anticancer Research, vol. 19, no. 4, pp. 2415-2420, 1999.

[34] Y. Kagebayashi, K. Yoshida, T. Kitauchi et al., "Nuclear matrix protein 22 levels in patients with renal transplantation," Japanese Journal of Urology, vol. 89, no. 9, pp. 774-779, 1998.

[35] T. Ishiguro, M. Nakajima, M. Naito, T. Muto, and T. Tsuruo, "Identification of genes differentially expressed in B16 murine melanoma sublines with different metastatic potentials," Cancer Research, vol. 56, no. 4, pp. 875-879, 1996.

[36] T. Ishiguro, H. Nagawa, M. Naito, and T. Tsuruo, "Analysis of novel metastasis-associated gene TI-227," Japanese Journal of Cancer Research, vol. 91, no. 4, pp. 390-394, 2000.

[37] J. M. Myers-Irvin, D. Landsittel, and R. H. Getzenberg, "Use of the novel marker BLCA-1 for the detection of bladder cancer," Journal of Urology, vol. 174, no. 1, pp. 64-68, 2005.

[38] T. S. Van Le, J. Myers, B. R. Konety, T. Barder, and R. H. Getzenberg, "Functional characterization of the bladder cancer marker, BLCA-4," Clinical Cancer Research, vol. 10, no. 4, pp. 1384-1391, 2004.

[39] T. Oikawa, "ETS transcription factors: possible targets for cancer therapy," Cancer Science, vol. 95, no. 8, pp. 626-633, 2004.

[40] G. Buchwalter, C. Gross, and B. Wasylyk, "Ets ternary complex transcription factors," Gene, vol. 324, no. 1-2, pp. 1-14, 2004.

[41] J. M. Myers-Irvin, T. S. Van Le, and R. H. Getzenberg, "Mechanistic analysis of the role of BLCA-4 in bladder cancer pathobiology," Cancer Research, vol. 65, no. 16, pp. 7145-7150, 2005.

[42] G. N. Thalmann, B. Dewald, M. Baggiolini, and U. E. Studer, "Interleukin-8 expression in the urine after bacillus CalmetteGuerin therapy: a potential prognostic factor of tumor recurrence and progression," Journal of Urology, vol. 158, no. 4, pp. 1340-1344, 1997.

[43] M. Chikazawa, K. Inoue, S. Fukata, T. Karashima, and T. Shuin, "Expression of angiogenesis-related genes regulates different steps in the process of tumor growth and metastasis in human urothelial cell carcinoma of the urinary bladder," Pathobiology, vol. 75, no. 6, pp. 335-345, 2008.

[44] B. M. Mian, C. P. N. Dinney, C. E. Bermejo et al., "Fully human anti-interleukin 8 antibody inhibits tumor growth in orthotopic bladder cancer xenografts via down-regulation of matrix metalloproteases and nuclear factor- $\kappa \mathrm{B}$," Clinical Cancer Research, vol. 9, no. 8, pp. 3167-3175, 2003.

[45] S. Tseng-Rogenski and M. Liebert, "Interleukin-8 is essential for normal urothelial cell survival," American Journal of Physiology, vol. 297, no. 3, pp. F816-F821, 2009.

[46] D. K. Ahirwar, A. Mandhani, and R. D. Mittal, "IL-8 -251 T > a polymorphism is associated with bladder cancer susceptibility and outcome after BCG immunotherapy in a Northern indian cohort," Archives of Medical Research, vol. 41, no. 2, pp. 97$103,2010$.

[47] C. Escudero-Lourdes, T. Wu, J. M. Camarillo et al., "Interleukin-8 (IL-8) over-production and autocrine cell activation are key factors in monomethylarsonous acid [MMA(III)]induced malignant transformation of urothelial cells," Toxicology and Applied Pharmacology, vol. 258, pp. 10-18, 2012.

[48] C. Feng, M. Guan, Q. Ding et al., "Expression of pigment epithelium-derived factor in bladder tumour is correlated with interleukin-8 yet not with interleukin-1 $\alpha$," Journal of Huazhong University of Science and Technology, vol. 31, no. 1, pp. 21-25, 2011.

[49] M. Seddighzadeh, P. Larsson, A. C. Ulfgren et al., "Low IL$1 \alpha$ expression in bladder cancer tissue and survival," European Urology, vol. 43, no. 4, pp. 362-368, 2003.

[50] C. T. Esmon, "Thrombomodulin as a model of molecular mechanisms that modulate protease specificity and function at the vessel surface," The FASEB Journal, vol. 9, no. 10, pp. 946-955, 1995.

[51] E. M. Conway, M. Van de Wouwer, S. Pollefeyt et al., "The lectin-like domain of thrombomodulin confers protection from neutrophil-mediated tissue damage by suppressing adhesion molecule expression via nuclear factor $\kappa \mathrm{B}$ and mitogen-activated protein kinase pathways," Journal of Experimental Medicine, vol. 196, no. 5, pp. 565-577, 2002.

[52] C. T. Esmon and W. G. Owen, "The discovery of thrombomodulin," Journal of Thrombosis and Haemostasis, vol. 2, no. 2, pp. 209-213, 2004.

[53] H. C. Huang, G. Y. Shi, S. J. Jiang et al., "Thrombomodulinmediated cell adhesion: involvement of its lectin-like domain," Journal of Biological Chemistry, vol. 278, no. 47, pp. 4675046759, 2003.

[54] R. D. Rosenberg and J. S. Rosenberg, "Natural anticoagulant mechanisms," Journal of Clinical Investigation, vol. 74, no. 1, pp. 1-6, 1984.

[55] B. R. Konety, T. S. T. Nguyen, R. Dhir et al., "Detection of bladder cancer using a novel nuclear matrix protein, BLCA-4," Clinical Cancer Research, vol. 6, no. 7, pp. 2618-2625, 2000.

[56] T.-S. Van Le, R. Miller, T. Barder, M. Babjuk, D. M. Potter, and R. H. Getzenberg, "Highly specific urine-based marker of bladder cancer,” Urology, vol. 66, no. 6, pp. 1256-1260, 2005. 


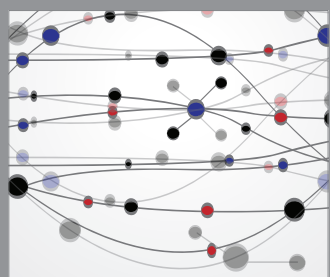

The Scientific World Journal


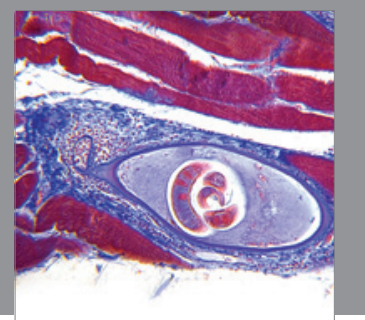

Gastroenterology

Research and Practice
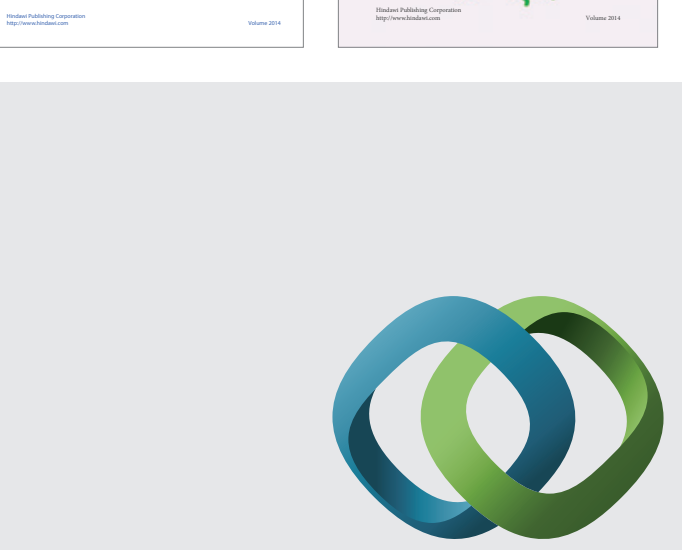

\section{Hindawi}

Submit your manuscripts at

http://www.hindawi.com
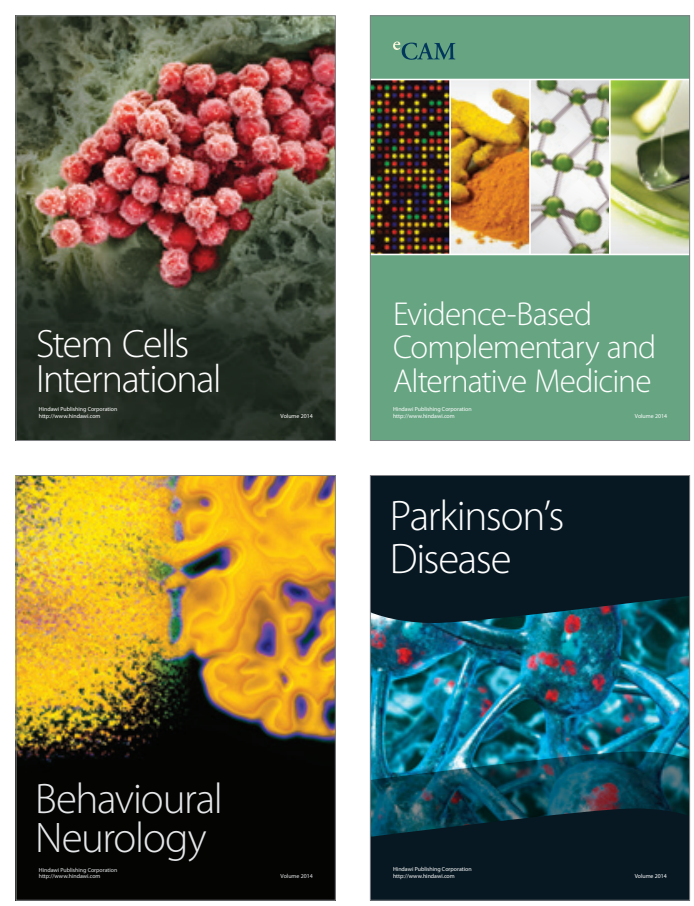

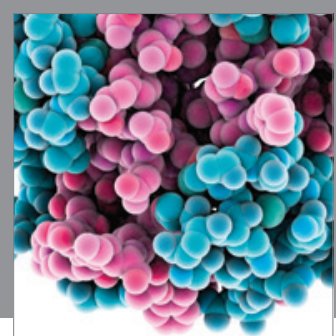

Journal of
Diabetes Research

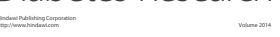

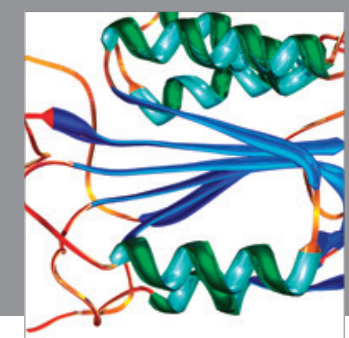

Disease Markers
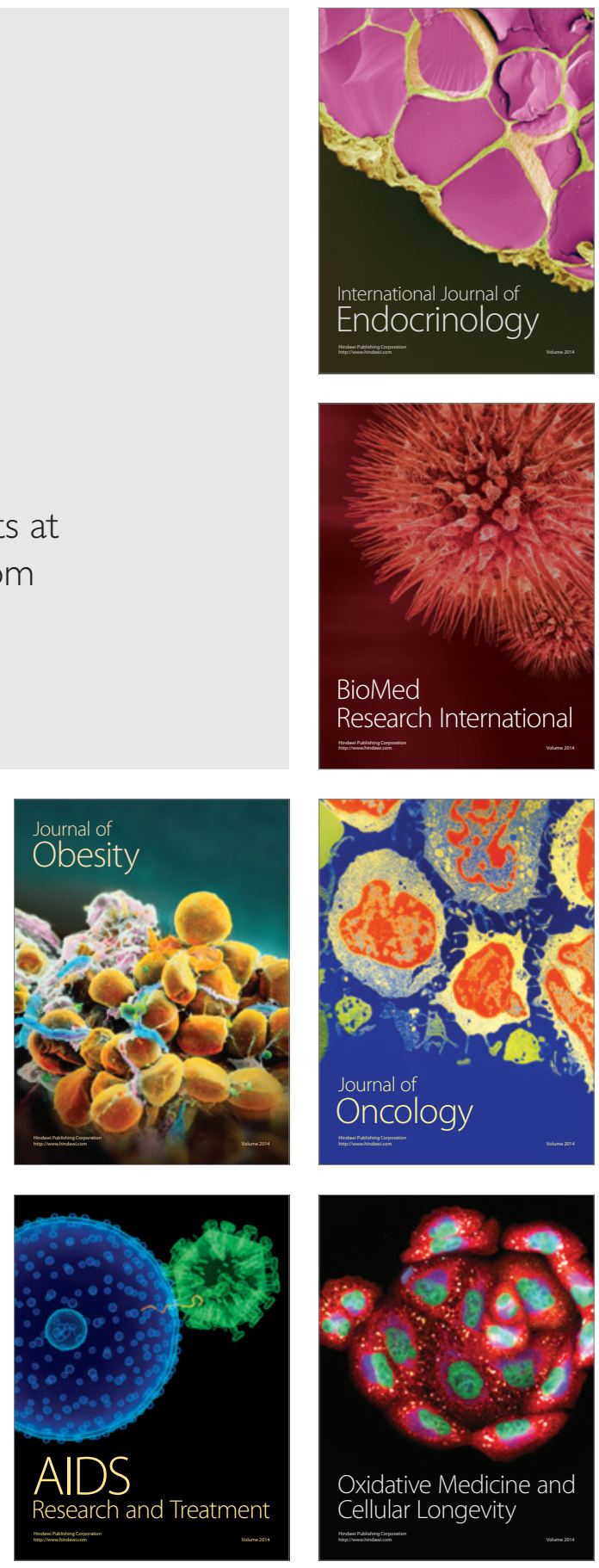